\title{
Structure and Dynamics of Magnetized Dark Molecular Clouds
}

\author{
P. S. $\mathbf{L i}^{1}$, C. F. McKee ${ }^{1,2}$ and R. I. Klein ${ }^{1,3}$
}

1Astronomy Department, University of California, Berkeley, CA 94720, USA email: psli@berkeley.edu

2Physics Department, University of California, Berkeley, CA 94720, USA

3Lawrence Livermore National Laboratory, P.O. Box 808, L-23, Livermore, CA 94550, USA

Massive infrared dark clouds (IRDCs) are believed to be the precursors to star clusters and massive stars (e.g. Bergin \& Tafalla 2007). The supersonic, turbulent nature of molecular clouds in the presence of magnetic fields poses a great challenge in understanding the structure and dynamics of magnetized molecular clouds and the star formation therein. Using the high-order radiation-magneto-hydrodynamic adaptive mesh refinement (AMR) code ORION2 ( $\mathrm{Li}$ et al. 2012), we perform a large-scale driven-turbulence simulation to reveal the 3D filamentary structure and dynamical state of a highly supersonic (thermal Mach number $=10$ ) and strongly magnetized (plasma $\beta=0.02$ ) massive infrared dark molecular cloud. With the high resolution afforded by AMR, we follow the dynamical evolution of the cloud in order to understand the roles of strong magnetic fields, turbulence, and self-gravity in shaping the cloud and in the formation of dense cores.

The most massive dark cloud filament at half a free-fall time in the simulation has a length of about $4-5 \mathrm{pc}$ with a mass of about $600-650 M_{\odot}(\sim 20 \%$ of the total mass in the simulated region). The mean density $n(\mathrm{H})$ and column density $N(\mathrm{H})$ are $\sim 2 \times 10^{4} \mathrm{~cm}^{-3}$ and $\sim 4 \times 10^{22} \mathrm{~cm}^{-2}$, respectively. The velocity dispersion is $1 \sim 1.5 \mathrm{~km}$ $\mathrm{s}^{-1}$. We observe a very complex magnetic field structure around the cloud filament, with most magnetic field lines piercing through the dark cloud filament in a direction roughly normal to the filament axis, consistent with polarization observations of filamentary IRDCs (e.g. Pereyra \& Magalhães 2004, Alves et al. 2008). The column density profiles of the cloud filament are well fitted by the power law $N \propto r^{-k_{\rho}}$ with $k_{\rho} \sim 0.6-0.8$, as observations have found (e.g. Hill et al. 2011, Arzoumanian et al. 2011). We use RADMC3D (Dullemond 2012) to generate molecular line emission images. Emission line profiles show both single and multiple line peaks and appear to be consistent with the braided filaments observed in dark clouds (e.g. Moriarty-Schieven et al. 1997, Hill et. al. 2011). We also observe several gravitationally collapsing cores that show a disklike structure with aspect ratios as high as $4-5$. The maximum density-weighted line-of-sight, the volume-weighted root-mean-square, and the highest magnetic field strength in the dense cores in our simulation are about $0.2,0.4$, and $5 \mathrm{mG}$, respectively.

\section{References}

Alves, F. O., Franco, G. A. P., \& Girart, J. M. 2008, A\&A 486, 13

Arzoumanian, D., Andre, P., Didelon, P., Konyves, V., Schneider, N., et al. 2011, A\&\&A 529, L6

Bergin, E. A. \& Tafalla, M. 2007, ARAA 45, 339

Dullemond, C. P. 2012 http://www.ita.uni-heidelberg.de/ dullemond/software/radmc-3d

Hill, T., Motte, F., Didelon, P., Bontemps, S., Minier, V., et al. 2011, A\&A 533, A94

Li, P. S., Martin, D., Klein, R. I., \& McKee, C. F. 2012, ApJ 745, 139

Moriarty-Schieven, G. H., Anderson, B. G., \& Wannier, P. G. 1997, ApJ 475, 642

Pereyra, A. \& Magalhães, A. M. 2004, ApJ 603, 584 